\title{
Effects of weight loss on asthma control in obese patients with severe asthma
}

\author{
Sérvulo Azevedo Dias-Júnior ${ }^{1}$, Monica Reis², Regina Maria de Carvalho-Pinto', \\ Rafael Stelmach ${ }^{1}$, Alfredo Halpern ${ }^{2}$ and Alberto Cukier ${ }^{1}$
}

Affiliations: ${ }^{1}$ Pulmonary Division, Heart Institute (InCor), Hospital das Clínicas da Faculdade de Medicina da Universidade de São Paulo, São Paulo, and ${ }^{2} O$ besity and Metabolic Syndrome Group, Endocrinology Service, Hospital das Clínicas da Faculdade de Medicina da Universidade de São Paulo, São Paulo, Brazil.

Correspondence: A. Cukier, Pulmonary Division, Instituto do Coração, Av. Dr. Enéas de Carvalho Aguiar, 44, Quinto andar, CEP 05403-900, São Paulo, SP, Brazil. E-mail: pnealbertodincor.usp.br

ABSTRACT Studies on the effects of weight loss in patients with asthma are scarce. No studies have been performed in patients with severe asthma. Therefore, the aim of the present study was to assess the impact of weight loss in patients with severe asthma associated with obesity.

This was an open, prospective, randomised study of two parallel groups, in patients with severe uncontrolled asthma and moderate obesity. The primary outcome was the level of asthma control 6 months after initiation of the weight reduction programme, quantified using the Asthma Control Questionnaire (ACQ). We evaluated clinical parameters, lung function, markers of airway inflammation and circulating cytokines.

22 patients were randomised to undergo treatment for obesity and 11 to the control group. The weight reduction programme was associated with significant improvements in asthma control (mean \pm SE ACQ score $3.02 \pm 0.19$ to $2.25 \pm 0.28$ in the treatment group versus $2.91 \pm 0.25$ to $2.90 \pm 0.16$ in the controls, $\mathrm{p}=0.001$ ). This improvement was not accompanied by changes in markers of airway inflammation or bronchial reactivity, but by an increase in forced vital capacity.

Our results suggest that weight reduction in obese patients with severe asthma improves asthma outcomes by mechanisms not related to airway inflammation.

@ERSpublications

Weight loss improves outcomes in severely asthmatic obese patients; poor control results from obesity-related factors http://ow.ly/qtG7w

This article has supplementary material available from www.erj.ersjournals.com

Received: March 232013 | Accepted after revision: Oct 232013 | First published online: Nov 142013

Clinical trial: This study is registered at www.ClinicalTrials.gov with identifier number NCT01049657.

Support statement: The study was funded by the FAPESP (grant number 08/55173-9).

Conflict of interest: Disclosures can be found alongside the online version of this article at www.erj.ersjournals.com

Copyright @ERS 2014 


\section{Introduction}

Severe asthma affects $<10 \%$ of asthmatic patients but has a disproportionate impact on the use of health resources, contributing to at least half of the direct and indirect costs of the disease $[1,2]$. An important component of the management of these patients is the correct diagnosis and treatment of comorbid or associated conditions that may influence asthma control.

The proportion of obese or overweight individuals is elevated in patients with severe asthma [3]. In the TENOR (The Epidemiology and Natural History of Asthma: Outcomes and Regional Treatment Regimens) study [3], the mean body mass index (BMI) of adult patients was $30.4 \mathrm{~kg} \cdot \mathrm{m}^{-2}$. More than half of patients with severe asthma treated at our centre are obese [4]. In fact, obesity is a risk factor for asthma [5], and is associated with the severity of the disease [6], a poor response to corticosteroids [7, 8] and worse clinical control $[9,10]$ represented by more days with symptoms, more visits to the emergency department, greater use of rescue medication and greater loss of work days [6].

Several hypotheses have been proposed to explain the relationship between obesity and asthma severity and control [11]. First, obesity has unfavourable mechanical effects on lung function. Second, it is associated with a number of comorbid factors that might interfere with asthma control, such as dietary intake, decreased physical activity, gastro-oesophageal reflux and obstructive sleep apnoea. A further possible mechanism relates to the chronic, mild systemic inflammation associated with obesity potentially increasing airway inflammation.

Studies on the effects of weight loss in patients with asthma are still scarce. In a systematic review [11], only five out of 15 studies considered relevant by the authors reported asthma markers as outcomes. The only randomised controlled trial found, conducted in subjects with mild-to-moderate asthma, reported improvement in pulmonary function parameters, fewer asthma symptoms and improved quality of life in those who lost weight with clinical treatment [11]. More recently, DiXON et al. [12] prospectively investigated the role of loss of weight induced by bariatric surgery in the pathophysiology of asthma. They showed that the weight reduction was associated with better clinical control without changes in the cellularity of bronchoalveolar lavage, but with decreased airway responsiveness in those who had normal levels of IgE. None of these studies included patients with severe asthma.

The present study was designed to assess the impact of weight loss with a medical weight loss programme in patients with severe asthma associated with obesity. Our hypothesis was that weight reduction would result in better asthma control. To test it, we conducted an open, prospective, randomised study of two parallel groups, of 6 months duration, that evaluated the effects of a weight reduction programme on clinical parameters, as well as lung function, markers of airway inflammation, and circulating cytokines in patients with severe asthma and moderate obesity.

\section{Methods \\ Setting and participants}

This was a prospective, open study with two randomised, parallel groups. The participants were obese (BMI $\geqslant 30 \mathrm{~kg} \cdot \mathrm{m}^{-2}$ ) with severe asthma (online supplementary material). The local institutional review board (Hospital das Clínicas da Faculdade de Medicina da Universidade de São Paulo, Sao Paulo, Brazil) approved this study and all patients provided written informed consent. The study is registered on ClinicalTrials.gov as NCT 01049657.

\section{Design overview}

The patients were selected to participate in the study if their asthma was not controlled according to GINA (Global Initiative for Asthma) criteria [13] after a 3-month run-in period, during which medication adherence was checked, and the influence of exogenous or endogenous aggravating factors was evaluated. Eligible patients were randomised by computer-generated randomisation in blocks in a $2 / 1$ ratio (weight loss/control).

The weight-loss programme consisted of low caloric intake, and use of sibutramine (10 mg per day) (patients with any cardiovascular risk were excluded) and orlistat (maximum dose of $120 \mathrm{mg}$ per day). The principles and behaviour methods of this weight reduction programme are described elsewhere [14]. All participants attended bimonthly consultations in the asthma clinic, where they were seen by the same investigator (S.A. Dias-Júnior), had the use of their inhaler checked, compliance with medication assessed by a count of the remaining doses, received the same amount of education about asthma [15], and were followed for 6 months. 


\section{Outcome measures}

The primary outcome measure was the level of asthma control 6 months after initiation of the weight reduction programme, quantified using the Asthma Control Questionnaire (ACQ) [16].

Secondary clinical outcomes included the Asthma Control Test (ACT) [17], lung function results, the St George's Respiratory Questionnaire (SGRQ) [18], change in methacholine reactivity (in participants with a forced expiratory volume in $1 \mathrm{~s}$ (FEV1) that was $\geqslant 50 \%$ predicted), daily use of asthma reliever medication, percentage of asthma symptom-free days, number of visits to the emergency department and exacerbations, and the Gastroesophageal Reflux Disease-Health-Related Quality-of-Life Scale [19].

Markers of airway cellular inflammation were measured in induced sputum [20] and with exhaled nitric oxide (NIOXMINO; Aerocrine AB, Solna, Sweden). IgE, C-reactive protein, leptin, eotaxin and transforming growth factor (TGF)- $\beta 1$ (ELISA; R\&D Systems Inc., Minneapolis, MN, USA) levels in serum were also measured.

\section{Statistical analysis}

The study was powered on the assumption that the ACQ score would show a difference of 0.5 between the groups. Assuming a Type I error rate of $0.05,11$ participants would provide $>80 \%$ power to detect a significant difference.

The intent-to-treat (ITT) population included all patients randomised who concluded the 6-month followup. The per protocol population (PP) was defined as the patients who lost $>10 \%$ of their body weight.

Data are presented as $\mathrm{n}(\%)$ for qualitative variables, mean \pm se for the variables normally distributed and median (interquartile range) for non-normally distributed data.

We used a t-test, Fisher's exact test or Mann-Whitney test to compare differences between the groups. Repeated-measures ANOVA was used to compare changes in measures from baseline to 6 months after initiation of the weight reduction programme. We studied the difference between study groups, the time effect, and the interaction between group and time effect. The Mann-Whitney test was used to compare between the groups and the Friedman test to compare within each group.

A descriptive level of $\mathrm{p}<0.05$ was adopted for all tests.

\section{Results}

\section{Baseline characteristics}

37 patients met the criteria for inclusion in the study; 33 were randomised (fig. 1). Of the 22 patients randomised to undergo treatment for obesity, 12 achieved the weight loss goal of $>10 \%$ of body weight.

The characteristics of patients at baseline are shown in table 1 and online supplementary table S1. The group consisted predominantly of females with moderate obesity and severe uncontrolled asthma, despite the use of high doses of controller medication (median dose of budesonide of $1600 \mu \mathrm{g}$ per day). Most patients reported that they were diagnosed with asthma in childhood or adolescence. They disclosed a functional pattern of moderate airflow obstruction, air trapping and increased airway resistance. The

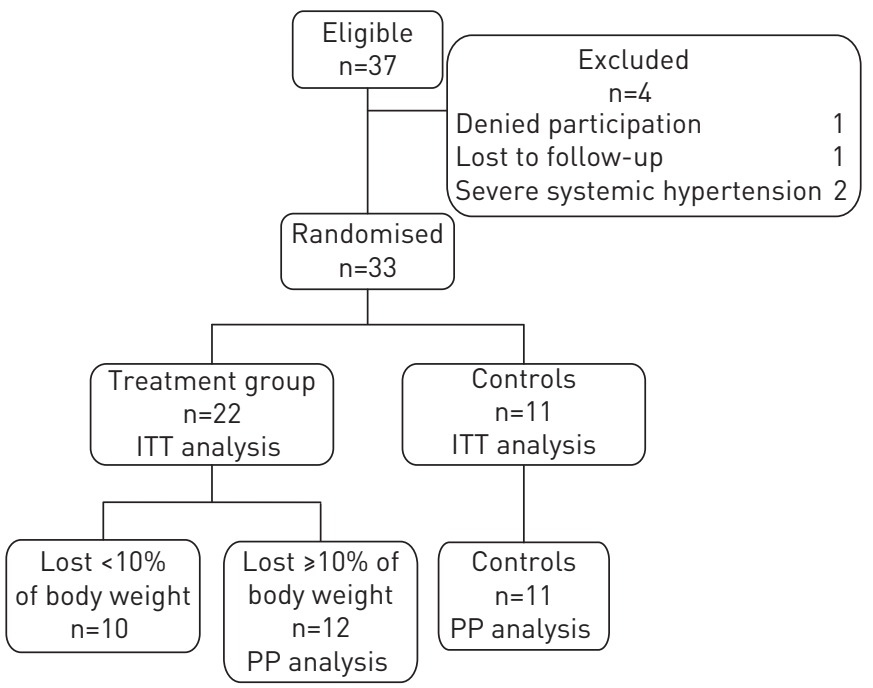

FIGURE 1 Enrolment and followup of study patients. ITT: intent-totreat; PP: per protocol. 
baseline values of inflammatory markers in sputum showed a marked eosinophilia. The increased serum levels of IgE were consistent with a predominance of atopic asthma.

\section{Effect of weight reduction on asthma control}

The average reduction in weight in the treatment group was $7.88 \mathrm{~kg}$, corresponding to $7.5 \%$ of baseline weight. BMI decreased significantly in the treatment group and remained unchanged in the control group. The ITT analysis also showed improvement in the control as measured by the ACQ, but did not detect changes in the ACT or SGRQ (table S2). Weight reduction in the PP analysis resulted in a statistically and clinically significant improvement in asthma control, measured by the ACQ, ACT and SGRQ (table 2).

There was symptomatic improvement in these individuals, represented by the increase in symptom-free days ( $p=0.022$, PP analysis), less use of rescue medication $(p=0.002$, PP analysis $)$ and fewer visits to the emergency room ( $p=0.0095, \mathrm{PP}$ analysis) during the study period. There were no differences in the number of exacerbations. The quality of life related to gastro-oesophageal reflux also did not vary.

ACQ reached clinically significant improvements $(>0.5)$ in 11 of the 12 patients who lost $>10 \%$ of their baseline weight, and in three of the 10 patients in the intervention group who did not reach the target weight loss (fig. 2).

\section{Effect of weight reduction on lung function tests}

Pulmonary function is shown in table 3 (and table S3). Forced vital capacity increased significantly in the treatment group and remained unchanged in the control group. Forced vital capacity was significantly improved in patients in the intervention group who lost $>10 \%$ of their baseline weight, but not in the patients who did not reach the target weigh loss (fig. 3). There was a trend toward a correlation between the change in ACQ score and the change in FVC $(r=-0.38, p=0.082)$. The other measures showed no differences between groups.

TABLE 1 Baseline demographic, clinical and pulmonary function data, and markers of inflammation and IgE level of study patients: intent-to-treat analysis

\begin{tabular}{|c|c|c|c|}
\hline Subjects $n$ & 11 & 22 & \\
\hline Females \% & 100 & 90.9 & $0.542^{\oplus}$ \\
\hline $\mathrm{BMI} \mathbf{k g} \cdot \mathrm{m}^{-2}$ & $37.29 \pm 1.07$ & $39.68 \pm 1.31$ & $0.243^{9}$ \\
\hline ACQ score & $2.91 \pm 0.25$ & $3.02 \pm 0.19$ & $0.718^{\bullet}$ \\
\hline Age of asthma onset years & $12(19)$ & $10(25)$ & $0.795^{\#}$ \\
\hline Rhinitis \% & 90.9 & 77.3 & $0.407^{\oplus}$ \\
\hline Gastro-oesophageal reflux disease $\%$ & 81.8 & 59.1 & $0.258^{\bullet}$ \\
\hline Smoking exposure pack-years & $3.86 \pm 1.12$ & $4.50 \pm 1.44$ & $0.437^{\circ}$ \\
\hline Budesonide dose $\mu \mathrm{g}$ per day & $1600 \overline{(800)}$ & $1600(0)$ & $0.985^{\#}$ \\
\hline Formoterol \% & 100 & 100 & $>0.999$ \\
\hline Omeprazole dose $\mathrm{mg}$ & $80(0)$ & $80(0)$ & $>0.999^{\#}$ \\
\hline TLC \% pred & $111.2 \pm 3.3$ & $106.5 \pm 3.8$ & 0.756 \\
\hline RV \% pred & $186.4 \pm 13.7$ & $153.0 \pm 8.4$ & 0.043 \\
\hline $\mathrm{RV} / \mathrm{TLC}$ & $0.53 \pm 0.03$ & $0.45 \pm 0.02$ & 0.006 \\
\hline Raw $\mathrm{cmH}_{2} \mathrm{O} \cdot \mathrm{L}^{-1} \cdot \mathrm{s}^{-1}$ & $4.69 \pm 0.58$ & $3.63 \pm 0.33$ & 0.177 \\
\hline FeNO ppb & $20.1 \pm 4.9$ & $25.1 \pm 3.1$ & 0.089 \\
\hline Induced sputum eosinophils \% & $11.9 \pm 3.9$ & $13.9 \pm 2.8$ & 0.489 \\
\hline Induced sputum neutrophils $\%$ & $41.7 \pm 4.0$ & $40.3 \pm 4.4$ & 0.450 \\
\hline Serum IgE IU $\cdot \mathrm{mL}^{-1}$ & $409.18 \pm 107.0$ & $382.8 \pm 56.7$ & 0.344 \\
\hline
\end{tabular}


TABLE 2 Clinical outcomes of study patients before and 6 months after weight reduction: per protocol analysis

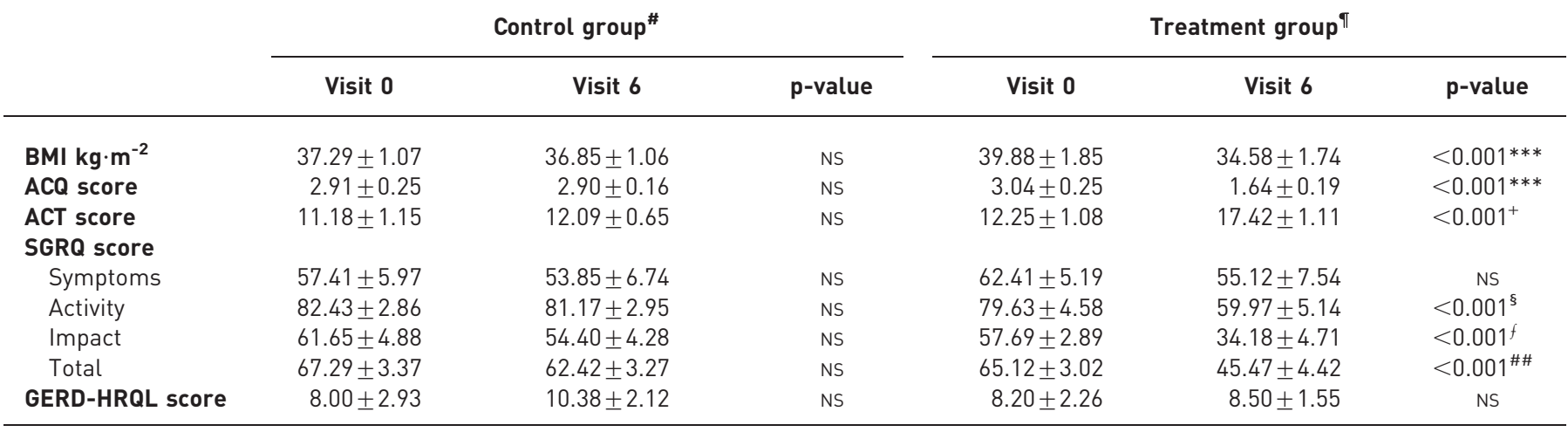

Data are presented as mean \pm SE unless otherwise stated. $p$-values were calculated by ANOVA. BMI: body mass index; ACQ: Asthma Control Questionnaire; ACT: Asthma Control Test; SGRQ: St George's Respiratory Questionnaire; GERD-HRQL: Gastroesophageal Reflux Disease-HealthRelated Quality-of-Life Scale; NS: nonsignificant. ${ }^{\#}: n=11 ;{ }^{\star}: \mathrm{n}=12 .{ }^{* * *}: \mathrm{p}<0.001$ between groups; ${ }^{+}: \mathrm{p}=0.024$ between groups; ${ }^{\S}: \mathrm{p}=0.005$ between groups; ${ }^{f}: p=0.028$ between groups; ${ }^{\# \#}: p=0.011$ between groups.

Effect of weight reduction on airway hyperresponsiveness, and on markers of airway and systemic inflammation

Airway hyperresponsiveness, exhaled nitric oxide levels and induced sputum cellularity did not change throughout the study. Leptin levels decreased in both groups ( $\mathrm{p}=0.013$ baseline versus 6 months, independent of group). Serum levels of IgE, C-reactive protein, eotaxin, and TGF- $\beta 1$ did not change (table 4 and table S4).

\section{Discussion}

In this prospective trial conducted in obese patients with severe asthma, a weight reduction programme was associated with improvements in asthma control. This improvement was not accompanied by changes in markers of airway inflammation or bronchial reactivity, but by an increase in forced vital capacity. This result suggests that weight loss has an important role in the treatment of obese difficult-to-control asthmatic subjects.

Our results add to the published medical literature, demonstrating the importance of obesity and its treatment in the management of patients with severe asthma.

In this study, the group of patients included those who belonged to the low-income group of a developing country, was composed mainly of females with moderate obesity and early-onset severe asthma that was
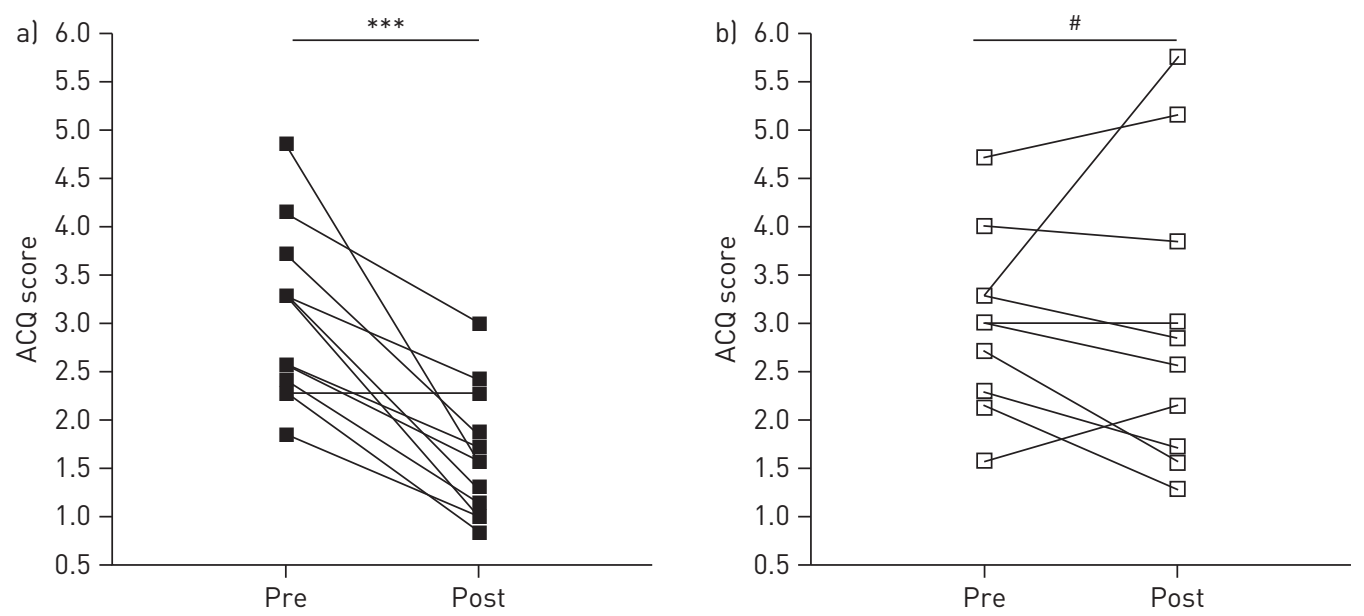

FIGURE 2 Impact of degree of weight loss on Asthma Control Questionnaire (ACQ) score. a) $>10 \%$ weight loss; b) $\leqslant 10 \%$ weight loss. ${ }^{\star}: \mathrm{p}<0.05 ;{ }^{* * *}: \mathrm{p}<0.001 ;{ }^{*}: \mathrm{p}=0.974$. 
TABLE 3 Pulmonary function of study patients before and 6 months after weight reduction: per protocol analysis

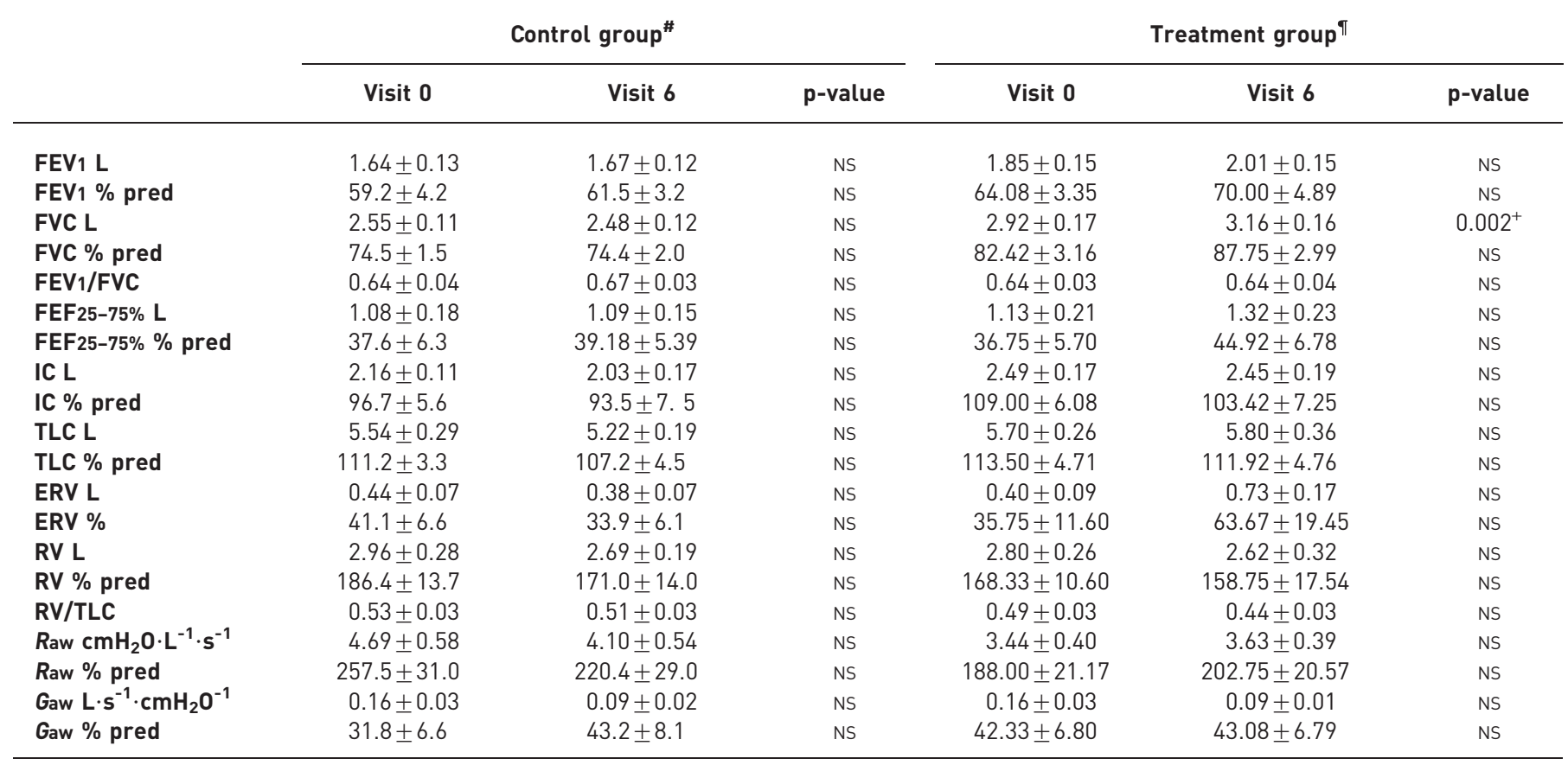

Data are presented as mean \pm SE unless otherwise stated. p-values were calculated by ANOVA. FEV1: forced expiratory volume in the $1 \mathrm{~s}$; FVC: forced vital capacity; FEF25-75\%: forced expiratory flow at 25-75\% of FVC; IC: inspiratory capacity; TLC: total lung capacity; ERV: expiratory reserve volume; RV: residual volume; Raw: airway resistance; Gaw: airway conductance; NS: nonsignificant. ${ }^{\#}: n=11 ;{ }^{\oplus}: n=12 ;{ }^{+}: p=0.006$ between groups.

markedly uncontrolled despite high doses of maintenance medication, with decreased pulmonary function, high morbidity, poor health-related quality of life, high levels of serum IgE and eosinophilic airway inflammation. Several authors [21-24] have suggested that obesity-associated asthma is different from asthma found in nonobese patients. Cluster analysis in a USA cohort of severe asthma patients [24] identified a group of mostly older obese females with late-onset nonatopic asthma, moderate reductions in FEV1 and frequent oral corticosteroid use to manage exacerbations. In their analysis of a dataset of patients with a diagnosis of refractory asthma in the UK, HALDAR et al. [23] described an obese subgroup with a female preponderance, evidence of asthma symptoms and an absence of eosinophilic airway inflammation. Others did not find direct evidence of an airway inflammatory profile that is specific to obesity [25]. The patients included in our study best fit the early-onset atopic asthma type, with greater airway dysfunction, symptoms and eosinophilic airway inflammation on a high dose of corticosteroid [23]. We speculate that

FIGURE 3 Impact of degree of weight loss on forced vital capacity (FVC). ${ }^{*}: \mathrm{p}<0.05 ;{ }^{\#}: \mathrm{p}=0.546$.

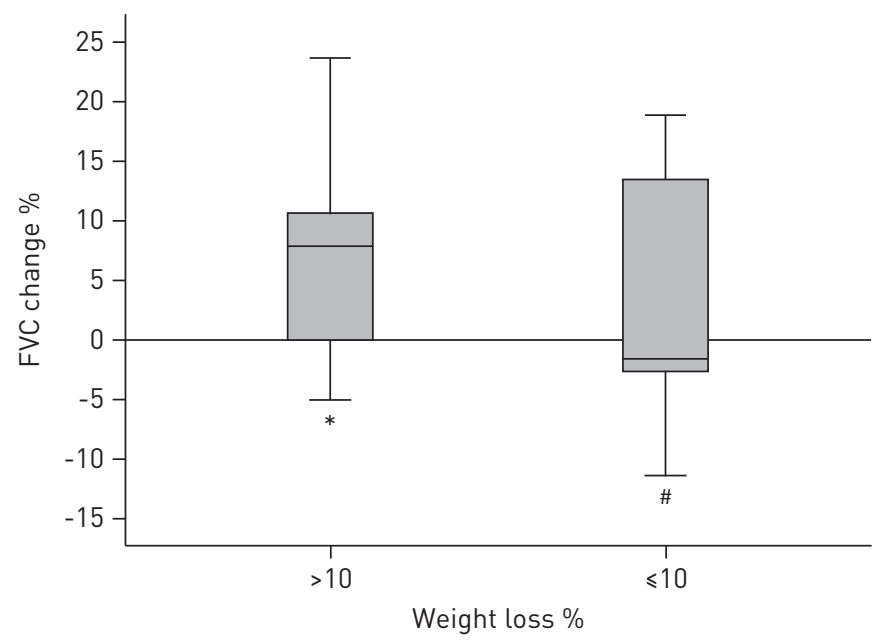


TABLE 4 Airway responsiveness, markers of inflammation and IgE level of study patients before and after weight reduction: per protocol analysis

\begin{tabular}{|c|c|c|c|c|c|c|}
\hline & \multicolumn{3}{|c|}{ Control group ${ }^{\#}$} & \multicolumn{3}{|c|}{ Treatment group $\pi$} \\
\hline & Visit 0 & Visit 6 & p-value & Visit 0 & Visit 6 & p-value \\
\hline FeNO ppb & $20.1 \pm 4.9$ & $19.3 \pm 3.2$ & NS & $19.6 \pm 3.7$ & $26.8 \pm 5.2$ & NS \\
\hline $\begin{array}{r}\text { Induced sputum } \\
\text { eosinophils \% }\end{array}$ & $11.9 \pm 3.9$ & $12.5 \pm 3.2$ & NS & $15.0 \pm 4.9$ & $14.4 \pm 4.7$ & NS \\
\hline $\begin{array}{l}\text { Induced sputum } \\
\text { lymphocytes \% }\end{array}$ & $3.0(4.0)$ & $3.0(4.0)$ & $N S^{\S}$ & $2.0(4.0)$ & $2.0(2.0)$ & $\mathrm{NS}^{\S}$ \\
\hline $\begin{array}{l}\text { Induced sputum } \\
\text { macrophages } \%\end{array}$ & $20.3 \pm 4.6$ & $12.5 \pm 3.4$ & NS & $17.4 \pm 3.1$ & $17.3 \pm 3.9$ & NS \\
\hline $\begin{array}{l}\text { Induced sputum } \\
\text { epithelial cells \% }\end{array}$ & $22.4 \pm 5.9$ & $17.7 \pm 5.3$ & NS & $24.4 \pm 6.5$ & $23.8 \pm 6.3$ & NS \\
\hline $\begin{array}{l}\text { Serum eotaxin } \\
\mathrm{pg} \cdot \mathrm{mL}^{-1}\end{array}$ & $116.1 \pm 28.8$ & $118.15 \pm 23.86$ & NS & $101.6 \pm 25.2$ & $106.1 \pm 23.4$ & NS \\
\hline TGF- $\beta 1 \mathrm{pg} \cdot \mathrm{mL}^{-1}$ & 2652.5 (311.7) & $2482.7(2688.6)$ & $N S^{\S}$ & $2620.8(287.8)$ & $2658.8(2529.1)$ & $\mathrm{NS}^{\S}$ \\
\hline
\end{tabular}

Data are presented as median (interquartile range) or mean \pm SE, unless otherwise stated. $p$-values were calculated by ANOVA. PD20: provocative dose of methacholine causing a $20 \%$ fall in forced expiratory volume in $1 \mathrm{~s}$ (FEV1); FeNO: exhaled nitric oxide fraction; TGF: transforming growth factor; NS: nonsignificant. ${ }^{\#}: \mathrm{n}=11 ;{ }^{\uparrow}: \mathrm{n}=12 ;^{+}: \mathrm{n}=10$ in the treatment group (two patients had FEV $1<50 \%$ predicted). ${ }^{\S}$ : Mann-Whitney test.

the phenotype described from the database of patients from developed countries, particularly the most severe, may not be universally applicable in scenarios of patients from a different background.

To the best of our knowledge, there are no previous prospective, randomised studies showing the benefit of a medical weight loss programme on asthma outcomes in obese, severely asthmatic patients such as those included in this study. In their meta-analysis of studies on weight loss and asthma in obese patients, ENELI et al. [11] found three observational studies and only one randomised trial that used low-calorie diets or a medical weight loss programme, all in patients with mild or moderate asthma. STENIUS-AARNiALA et al. [26] conducted a 1-year randomised controlled trial using low-calorie diets and reported that weight reduction improved lung function, symptoms, morbidity and health status. Patients with severe asthma were excluded from the study. In regard to bariatric surgery for asthma patients, DiXON et al. [12] reported that asthmatic participants had significant improvements in asthma control and asthma quality of life.

The statistically and clinically significant improvement in asthma control measured by the ACQ found in our study was not observed in recently published observational studies of patients with refractory or difficult-to-control asthma. BAFADHEL et al. [27] followed 129 patients with refractory asthma for 12 months, of whom $74 \%$ of patients had an elevated BMI (with characteristics very similar to those of our patients) and $77 \%$ of patients had a change in weight of $\pm 2 \mathrm{~kg}$ after 1 year. $54 \%$ of patients had a mean \pm SD increase in weight of $4.9 \pm 2.9 \mathrm{~kg}$ and $23 \%$ of patients had a decrease in weight of $4.4 \pm 2.8 \mathrm{~kg}$. Compared to baseline, there was no difference after 1 year in lung function, maintenance corticosteroid, inhaled corticosteroid dosage, ACQ scores, or eosinophilic airway inflammation in males or females who lost weight, gained weight or did not change their weight. HASELKORN et al. [28] analysed the effects of weight change on asthma control, asthma-related quality of life, number of steroid bursts and exacerbation of asthma symptoms in a population of adult patients with severe or difficult-to-treat asthma who participated in the TENOR study. Asthma patients who gained $>5 \mathrm{lb}(2.27 \mathrm{~kg})$ during the 12 -month interval between baseline and follow-up reported poorer asthma control, worse quality of life and a greater number of steroid bursts than patients who maintained their baseline weight or lost $<5 \mathrm{lb}$. The study did not identify improved outcomes with weight loss. The authors explain that their choice of the 5-lb cut off was the largest weight cut off they could use while making estimates of the effects with reasonable precision. In addition, 
$5 \mathrm{lb}$ was deemed to be a low, yet reasonable and actionable, cut point that physicians could easily communicate to their patients.

Getting patients to lose weight is undoubtedly a difficult task; only 12 out of 22 volunteers assigned to the treatment group in the present study reached the target loss of $>10 \%$ of the baseline weight. In these patients, the mean weight loss of $14.04 \mathrm{~kg}(13.3 \%$ of baseline weight $)$ was accompanied by a statistically and clinically significant decrease of 1.40 in the ACQ score (PP analysis), compared with a mean reduction in weight of $7.88 \mathrm{~kg}$ ( $7.5 \%$ of baseline weight), resulting in a decrease of 0.77 in the ACQ score in the ITT analysis of the 22 patients allocated to the treatment group. Our results are in accordance with those of SCOTT et al. [29], who suggest a weight loss goal of $5-10 \%$ be recommended to assist in the clinical management of overweight and obese adults with asthma. Taking into account our results and those of other authors, it is reasonable to conclude that obese patients with asthma of any severity will improve asthma control provided they lose an appropriate percentage of their baseline weight [26-29]. Considering that despite the improvement observed in our patients, their average ACQ score remained consistent with poor control $(>1.5)$; however, even those patients who lost $>10 \%$ of their weight remained obese. We can therefore speculate that reaching a normal weight would lead to asthma control in a substantial proportion of these patients.

Our study has some limitations. The nature of the intervention did not allow our study to remain blinded. The control group did not receive a placebo or control intervention, which could allow overestimation of the effect size. After randomisation, patients in the control group may have been disappointed, which could influence their assessment of symptoms and quality of life. Apart from the weight reduction programme, the control group received equal attention from the medical staff, as well as assured access to a weight reduction programme similar to that applied in the treatment group at study completion. The female predominance observed in our severe asthmatic population was previously reported [4]. The two males included in our study were randomised to the intervention group, reached the initial goal of $>10 \%$ reduction in weight and had clinically significant improvement in ACQ score, suggesting that the effects of weight loss can also be extended to males.

It can be argued that the persistent eosinophilia despite high doses of inhaled corticosteroids may result from nonadherence to treatment, particularly when only 12 of the 22 patients assigned to the treatment group were compliant with the weight loss intervention. We have previously described persistent sputum eosinophilia despite steroid treatment not related to any specific clinical characteristic or to the inhaled corticosteroid dosage, in a cohort of patients similar to the cohort of the present study [4]. This profile is in accordance with the results of AMELINK et al. [30], who recently reported sputum eosinophilia in patients with severe asthma, despite the use of inhaled corticosteroids at doses equivalent to $1000 \mu \mathrm{g}$ of fluticasone. In another study, the same group described a "severe eosinophilic inflammation-predominant" cluster in which sputum eosinophilia was present despite treatment with medium-to-high doses of inhaled corticosteroids (in 26\% of the cases, combined with maintenance of oral corticosteroids) [31]. Taken together, these data suggest that these patients characterise a specific asthma subphenotype. Whatever the explanation for this airway inflammation behaviour, it does not interfere with the main conclusion of our study, which indicates that weight reduction improves control in severe asthma patients by mechanisms independent of asthmatic inflammation.

Given the study design, it can be argued that the drugs used in the weight reduction programme had some action on the mechanisms of asthma. There are no reports in the literature of such effects or drug interaction with bronchodilators or inhaled corticosteroids.

We recognise that a structured programme of exercises is an integral part of the process of weight reduction. However, we have avoided deliberately encouraging patients to exercise to avoid including a confounding factor in the analysis of the results, given that aerobic exercise may interfere with the inflammatory response of the airways [32].

The results of this study are consistent with previous studies that suggest that poor asthma control in people who are obese is partially related to obesity related-factors, which are not reflected by traditional measures of asthma. In a recent published study, FARAH et al. [10] evaluated asthmatic patients at baseline and 12 weeks after high-dose inhaled corticosteroid treatment. They showed that at baseline the independent predictors of ACQ results were FEV1, exhaled nitric oxide and BMI. After treatment, targeted against airway inflammation but not obesity, all patients improved irrespective of their weight. BMI became the sole independent determinant of asthma control. Studies in refractory asthma led to similar conclusions. In a cross-sectional evaluation of 139 obese and nonobese patients with difficult-to-treat asthma, van VEEN et al. [22] concluded that obesity was inversely related to sputum eosinophils and exhaled nitric oxide, and positively associated with the presence of comorbid factors and reduced lung volumes. In their 1-year 
observational study, BAFADHEL et al. [27] showed that change in weight was not associated with change in airway inflammation. DiXON et al. [12] suggested that the influence of weight reduction on airway responsiveness might be related to IgE levels, because they observed improvement in airway responsiveness only in nonatopic patients.

Our study has clinical implications. We suggest that, in people who are obese and have severe asthma, increasing the doses of corticosteroids may not be the best therapeutic alternative for patients who maintain symptoms. Weight reduction in obese patients improves asthma control, at least in part by attenuating the negative effects on lung volumes. Therefore, weight loss should be considered as a strategy to improve asthma control in these patients.

In conclusion, our study adds information to the controversy about the impact of obesity and its treatment on asthma control. Our results suggest that weight reduction in obese patients with severe asthma improves asthma outcomes by mechanisms not related to airway inflammation and that poor asthma control in people who are obese is, at least in part, the result of obesity-related factors. Although the sample analysed in our study was small, we can argue that the therapeutic approach for obese patients with difficult-to-treat asthma should therefore be aimed at weight reduction as well as at optimizing anti-inflammatory treatment.

\section{Acknowledgements}

The authors thank C.S. Redigolo and R.A. de Oliveira Figueiredo for assistance with the statistical analysis, and L. Antonangelo, L. Angelini and M.M.P. Acencio for their collaboration in data collection (all Hospital das Clínicas da Faculdade de Medicina da Universidade de São Paulo, Sao Paulo, Brazil).

\section{References}

1 Serra-Batlles J, Plaza V, Morejon E, et al. Costs of asthma according to the degree of severity. Eur Respir J 1998; 12 : 1322-1326.

2 Sullivan SD, Rasouliyan L, Russo PA, et al. Extent, patterns, and burden of uncontrolled disease in severe or difficult-to-treat asthma. Allergy 2007; 62: 126-133.

3 Dolan CM, Fraher KE, Bleecker ER, et al. Design and baseline characteristics of The Epidemiology and Natural History of Asthma: Outcomes and Treatment Regimens (TENOR) study: a large cohort of patients with severe or difficult-to-treat asthma. Ann Allergy Asthma Immunol 2004; 92: 32-39.

4 Carvalho-Pinto RM, Cukier A, Angelini L, et al. Clinical characteristics and possible phenotypes of an adult severe asthma population. Respir Med 2012; 106: 47-56.

5 Beuther DA, Sutherland ER. Overweight, obesity, and incident asthma: a meta-analysis of prospective epidemiologic studies. Am J Respir Crit Care Med 2007; 175: 661-666.

6 Taylor B, Mannino D, Brown C, et al. Body mass index and asthma severity in the National Asthma Survey. Thorax 2008; 63: 14-20.

7 Boulet LP, Franssen E. Influence of obesity on response to fluticasone with or without salmeterol in moderate asthma. Respir Med 2007; 101: 2240-2247.

8 Sutherland ER, Goleva E, Strand M, et al. Body mass and glucocorticoid response in asthma. Am J Respir Crit Care Med 2008; 178: 682-687.

9 Mosen DM, Schatz M, Magid DJ, et al. The relationship between obesity and asthma severity and control in adults. J Allergy Clin Immunol 2008; 122: 507-511.

10 Farah CS, Kermode JA, Downie SR, et al. Obesity is a determinant of asthma control independent of inflammation and lung mechanics. Chest 2011; 140: 659-666.

11 Eneli IU, Skybo T, Camargo CA. Weight loss and asthma: a systematic review. Thorax 2008; 63: 671-676.

12 Dixon AE, Pratley RE, Forgione PM, et al. Effects of obesity and bariatric surgery on airway hyperresponsiveness, asthma control, and inflammation. J Allergy Clin Immunol 2011; 128: 508-515.

13 Bateman ED, Hurd SS, Barnes PJ, et al. Global strategy for asthma management and prevention: GINA executive summary. Eur Respir J 2008; 31: 143-178.

14 Halpern A, Pepe R, Monegaglia AP, et al. Efficacy and tolerability of the association of sibutramine and orlistat for six months in overweight and obese patients. J Obes 2010; 2010: 602537.

15 Angelini L, Robles-Ribeiro PG, Carvalho-Pinto RM, et al. Two-year evaluation of an educational program for adult outpatients with asthma. J Bras Pneumol 2009; 35: 618-627.

16 Leite M, Ponte EV, Petroni J, et al. Avaliação do questionário de controle da asma validado para uso no Brasil [Evaluation of the asthma control questionnaire validated for use in Brazil]. J Bras Pneumol 2008; 34: 756-763.

17 Nathan RA, Sorkness CA, Kosinski M, et al. Development of the asthma control test: a survey for assessing asthma control. J Allergy Clin Immunol 2004; 113: 59-65.

18 Sousa TC, Jardim JJ, Jones P. Validation of the Saint George's Respiratory Questionnaire in patients with chronic obstructive pulmonary disease in Brazil. J Bras Pneumol 2000; 26: 119-125.

19 Fornari F, Gruber AC, Lopes AB, et al. Questionário de sintomas na doença do refluxo gastroesofágico [Symptom's questionnaire for gastroesophageal reflux disease]. Arq Gastroenterol 2004; 41: 263-267.

20 Saraiva-Romanholo BM, Barnabé V, Carvalho ALI, et al. Comparison of three methods for differential cell count in induced sputum. Chest 2003; 124: 1060-1066.

21 Lessard A, Turcotte H, Cormier Y, et al. Obesity and asthma: a specific phenotype? Chest 2008; 134: 317-23.

22 van Veen IH, ten Brinke A, Sterk PJ, et al. Airway inflammation in obese and nonobese patients with difficult-totreat asthma. Allergy 2008; 63: 570-574.

23 Haldar P, Pavord ID, Shaw DE, et al. Cluster analysis and clinical asthma phenotypes. Am J Respir Crit Care Med 2008; 178: 218-224. 
24 Moore WC, Meyers DA, Wenzel SE, et al. Identification of asthma phenotypes using cluster analysis in the Severe Asthma Research Program. Am J Respir Crit Care Med 2010; 181: 315-323.

25 Todd DC, Armstrong S, D'Silva L, et al. Effect of obesity on airway inflammation: a cross-sectional analysis of body mass index and sputum cell counts. Clin Exp Allergy 2007; 37: 1049-1054.

26 Stenius-Aarniala B, Poussa T, Kvarnström J, et al. Immediate and long term effects of weight reduction in obese people with asthma: randomised controlled study. BMJ 2000; 320: 827-832.

27 Bafadhel M, Singapuri A, Terry S, et al. Body mass and fat mass in refractory asthma: an observational 1 year follow-up study. J Allergy (Cairo) 2010; 2010: 251758.

28 Haselkorn T, Fish J, Chipps BE, et al. Effect of weight change on asthma-related health outcomes in patients with severe or difficult-to-treat asthma. Respir Med 2009; 103: 274-283.

29 Scott HA, Gibson PG, Garg ML, et al. Dietary restriction and exercise improve airway inflammation and clinical outcomes in overweight and obese asthma: a randomized trial. Clin Exp Allergy 2013; 43: 36-49.

30 Amelink M, de Groot JC, de Nijs SB, et al. Severe adult-onset asthma: a distinct phenotype. J Allergy Clin Immunol 2013; 132: 336-341.

31 Amelink M, de Nijs SB, de Groot JC, et al. Three phenotypes of adult-onset asthma. Allergy 2013; 68: 674-680.

32 Mendes FAR, Almeida FM, Cukier A, et al. Effects of aerobic training on airway inflammation in asthmatic patients. Med Sci Sports Exercise 2011; 43: 197-203. 